\title{
Breast cancer related lymphedema and surgical treatment
}

\section{Byung-Joon Jeon, David W. Chang}

Section of Plastic and Reconstructive Surgery, Department of Surgery, University of Chicago Medicine and Biological Sciences, Chicago, IL, USA

Received: March 31, 2020

Revised: May 4, 2020

Accepted: May 7, 2020

Corresponding author:

Byung-Joon Jeon

Section of Plastic and

Reconstructive Surgery,

Department of Surgery,

University of Chicago Medicine

and Biological Sciences, $5758 \mathrm{~S}$.

Maryland Ave DCAM, Suite $4 \mathrm{H}$,

Chicago, IL 60637, USA

Tel: +1-312-420-8088

E-mail: saintmed@naver.com

\section{ABSTRACT}

Lymphedema is a chronic, progressive and debilitating disease. This review article focuses on surgical treatment of breast cancer-related lymphedema especially in perspectives of microsurgical options. In the early stage, where protein-rich fluid accumulates, expressed as pitting, conservative treatments, such as complex decongestive therapy and/or one of the physiologic treatments, lymphovenous bypass (LVB) can be considered. Once the stage is more advanced and skin fibrosis and adipose tissue deposition are appearing, it may be considered to attempt vascularized lymph node transplant (VLNT) alone or VLNT and LVB simultaneously. If the stage is more advanced and fibroadipose tissue accumulation is dominant, then debulking procedure such as liposuction may be considered. A direct excision may be needed for the most advanced stage of lymphedema.

Keywords: Breast neoplasms; Lymphedema; Therapeutics; Upper extremity
This is an Open Access article distributed under the terms of the Creative Commons Attribution Non-Commercial License (https:// creativecommons.org/licenses/ by-nc/4.0/).

\section{INTRODUCTION}

Lymphedema is the clinical manifestation of an impaired lymphatic system. The secondary or acquired lymphedema is more common than the primary one and usually happens after damage or obstruction of the previously healthy lymphatic system with the infection (e.g., filariasis), trauma, and various cancer treatments including lymphadenectomy, chemotherapy, and radiation therapy [1,2]. In most developed countries breast cancer-related lymphedema is the most common lymphedema associated with cancer treatment. The incidence has ranged from $14 \%$ to $40 \%$ with axillary lymph node dissection and sentinel-node biopsy reduced the risk to $6 \%$ to $10 \%[3,4]$. The risk of the breast cancer-related lymphedema depends on several factors, such as dissection of the axillary nodes, radiation therapy, postoperative complications, ipsilateral venous compromise, taxane-based chemotherapy, and the number of positive lymph nodes, especially more than eight $[4,5]$. This review article focuses on surgical treatment of breast cancer-related lymphedema especially in perspectives of microsurgical options. 


\section{LYMPHATIC ANATOMY}

The lymph fluid is made in the process of balancing hydrostatic and oncotic pressure in capillaries and tissues. The lymphatic system includes lymph nodes and lymphatic vessels. It has several interesting and unique characteristics. The lymphatic system, which begins with the blind tubes, passes through the lymph nodes and eventually connects to the heart through the veins, such as the subclavian vein. Unlike the veins, the lymphatics have fewer bifurcations, and they show the clear direction towards axillary or inguinal lymph nodes in the extremities and moves in one direction through the smooth muscle action and valves in the lymphatic vessels. It is also intriguing that the connection between the superficial and deep lymphatic system is not significant. Although there exist perforating lymphatic vessels and they connect the superficial and deep lymphatic vessels, some authors argue that they only parallel the arteries originating below the deep fascia and do not connect the two lymphatic systems definitely [6,7].

The lymphatic vessels can be largely divided into three categories including lymph capillaries, pre-collectors, and lymph-collecting vessels $[7,8]$. The lymph capillaries (20 to 70 $\mu \mathrm{m})$ are located just beneath the epidermis and have blind endings. They are formed with the endothelial cells that are loosely overlapped with each other. Anchoring filaments connect the endothelial cells and the surrounding tissue. In the case of excess interstitial fluid being, the anchoring filaments are stretched and the gaps between the endothelial cells are open to absorb the fluid [7,9]. The lymph capillaries don't have valves and are connected by the pre-collectors in the deep dermal layer.

The pre-collectors (70 to $150 \mu \mathrm{m}$ ) have unidirectional valves helping lymph flow from the superficial to deep layers and become larger vessels through meeting together in the dermal layer [10]. Via the efferent pre-collectors, they connect to the lymph-collecting vessels in the subcutaneous layers.

The lymph-collecting vessels ( 150 to $500 \mu \mathrm{m}$ ) can be subdivided as superficial and deep ones according to their anatomical location to the deep fascia. They have three-layered structure, including the endothelial, smooth muscle, and collagen fiber with fibroblast layers. The smooth muscle layer performs a major role in rhythmic contraction for the directional flow of the lymph fluid.

In the upper extremities, there may be an alternative pathway of the superficial lymph-collecting vessels that skips the axillary lymph nodes (sentry nodes) and goes to the supraclavicular lymph nodes through the deltopectoral nodes (in- terval lymph nodes). It can work as a detour for preventing upper-extremity lymphedema.

\section{DIAGNOSIS}

Edematous change of the upper extremities appears as an apparent symptom in the early stage, but fibroadipose tissue deposition becomes a dominant manifestation and a characteristic skin lesions (acanthosis, fat deposits, and warty overgrowths) are also present in the late stage. The detailed history and several laboratory tests may be needed for the differential diagnosis of lipedema, myxedema and an occult veno-occlusive disease [4].

In many cases, imaging studies are not mandatory for the exact diagnosis. But they can be helpful for the confirmative diagnosis and determining the treatment options.

Currently, lymphoscintigraphy is the definitive diagnostic test for lymphedema. This study involves the injection of a tracer protein (technetium Tc-99M ${ }^{99 \mathrm{~m}} \mathrm{Tc}$ ] sulfur colloid) into the webspace of the hand, after which the tracer is preferentially taken up by the lymphatics [11]. A gamma camera located over the patient then detects the ${ }^{99 \mathrm{~m}} \mathrm{Tc}$-sulfur colloid signal. Abnormal findings include delayed transit time to the regional lymph nodes, dermal backflow (accumulation of tracer in cutaneous lymphatics), asymmetric node uptake, and/or formation of a collateral lymphatic channel.

Near-infrared fluorescent lymphography is a relatively new imaging technique for assessing lymphatic system. The transit of dermally injected indocyanine green (ICG) dye is traced with fluorescent spectroscopy. This test is not only more sensitive than radionuclide lymphoscintigraphy in the early stages of lymphedema but also helpful to find the functional lymphatics for the physiologic lymphedema treatment $[12,13]$.

\section{Staging}

The lymphedema staging system plays an important role in describing the current status of the disease. Although there are various classification systems, a staging system for lymphedema, established by the International Society of Lymphology (ISL), is clinically based and well-known. The ISL system divides the lymphedema into four categories [14]. Stage 0 (or IA) refers to a latent or subclinical condition where swelling is not evident despite impaired lymph transport and changes in subjective symptoms. Stage I shows an early accumulation of fluid which subsides with limb elevation. Pitting may occur. Stage II signifies that limb elevation alone rarely reduces the tissue swelling and pitting is manifest. Later in stage II, the 
limb may not pit as excess subcutaneous fat and fibrosis develop. Stage III encompasses lymphostatic elephantiasis where pitting can be absent and trophic skin changes have developed. A practical staging system based on the findings with ICG lymphangiography was suggested by Chang et al. [15]. The system categorizes the lymphedema into four according to the pattern of dermal backflow and the numbers of lymphatics visualized $[15,16]$. Stage 1 shows minimal, patchy dermal backflow with many patent lymphatic vessels. Stage 2 refers to segmental dermal backflow with a moderate number of patent lymphatic vessels. Stage 3 presents extensive dermal backflow involving the entire arm and few patent lymphatic vessels. In stage 4, there exists severe dermal backflow involving the entire arm and extending to the dorsum of the hand and no patent lymphatic vessels are observed (Fig. 1).

\section{Treatment}

\section{Physiologic surgeries}

Surgical treatment of lymphedema can be grossly divided into physiologic surgery and debulking procedure. Physiologic surgery include lymphovenous bypass (LVB) and vascularized lymph node transplant (VLNT).

\section{1) Lymphovenous bypass}

LVB is an operation to connect the functioning lymphatics and the veins for transporting protein-rich fluid in the interstitial space bypassing damaged lymphatics, which is effective in the treatment of early-stage lymphedema, such as, ISL classification of lymphedema stage II or Chang's lymphedema stage 1 or 2. Therapeutic effects can be expected in more advanced lymphedema if functioning lymphatics can be found and the therapy with VLNT can be done simultaneously.

If the LVB is performed in an early stage of lymphedema, a decrease in volume and number of cellulitis may occur in addition to improvement of the subjective symptoms. Moreover, some patients may be less dependent on the compressive garments. The relatively low risk of complications is another advantage, and surgery can be carried out under the local anesthesia.
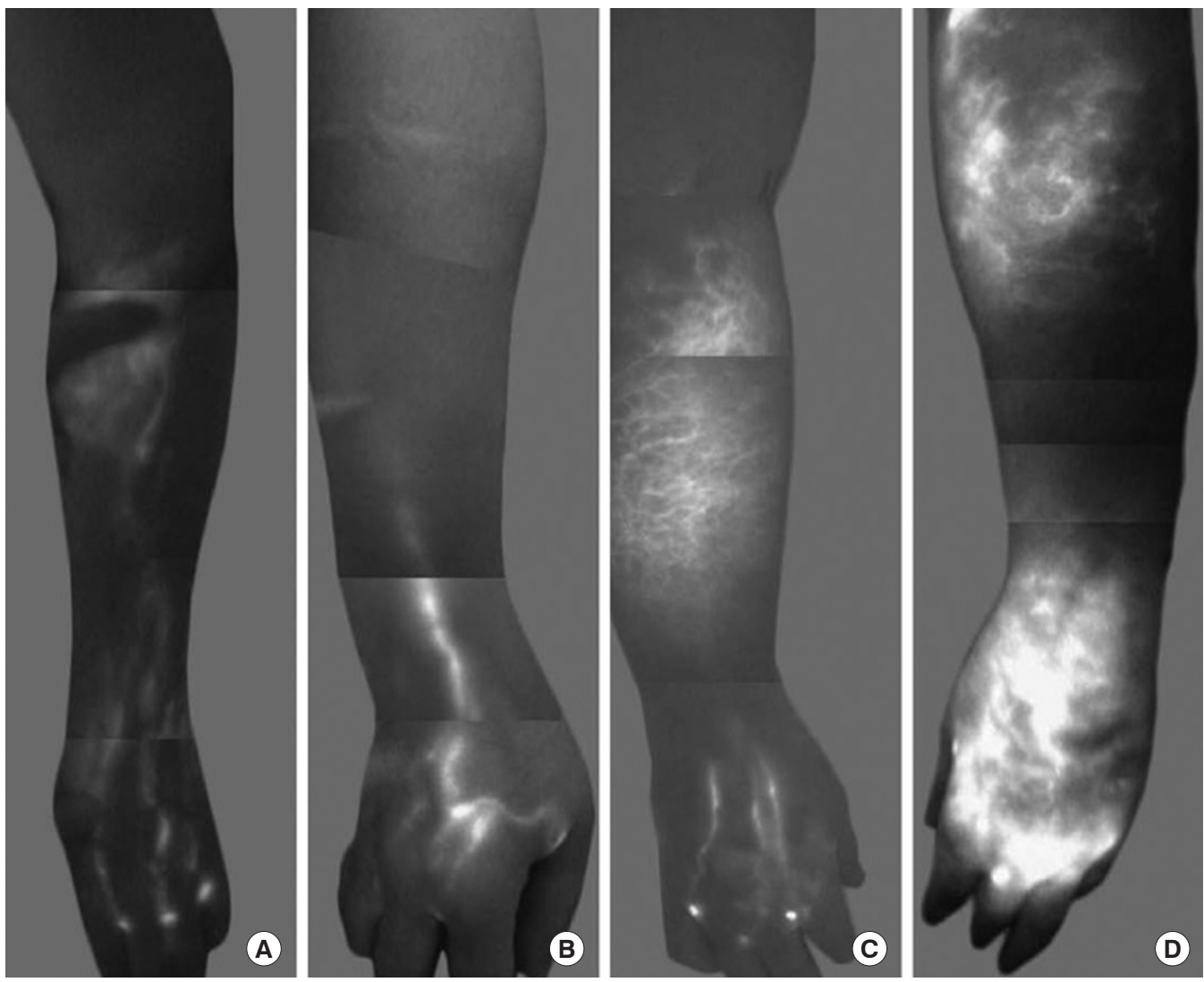

Fig. 1. MD Anderson lymphedema classification based on injected indocyanine green lymphangiographic findings. (A) Stage 1: many patent lymphatic vessels, with minimal, patchy dermal backflow. (B) Stage 2: moderate number of patent lymphatic vessels, with segmental dermal backflow. (C) Stage 3: few patent lymphatic vessels, with extensive dermal backflow involving the entire arm. (D) Stage 4: no patent lymphatic vessels seen with severe dermal backflow involving the entire arm and extending to the dorsum of the hand. Adapted from Chang et al. [15], with permission from Wolters Kluwer Health, Inc. 


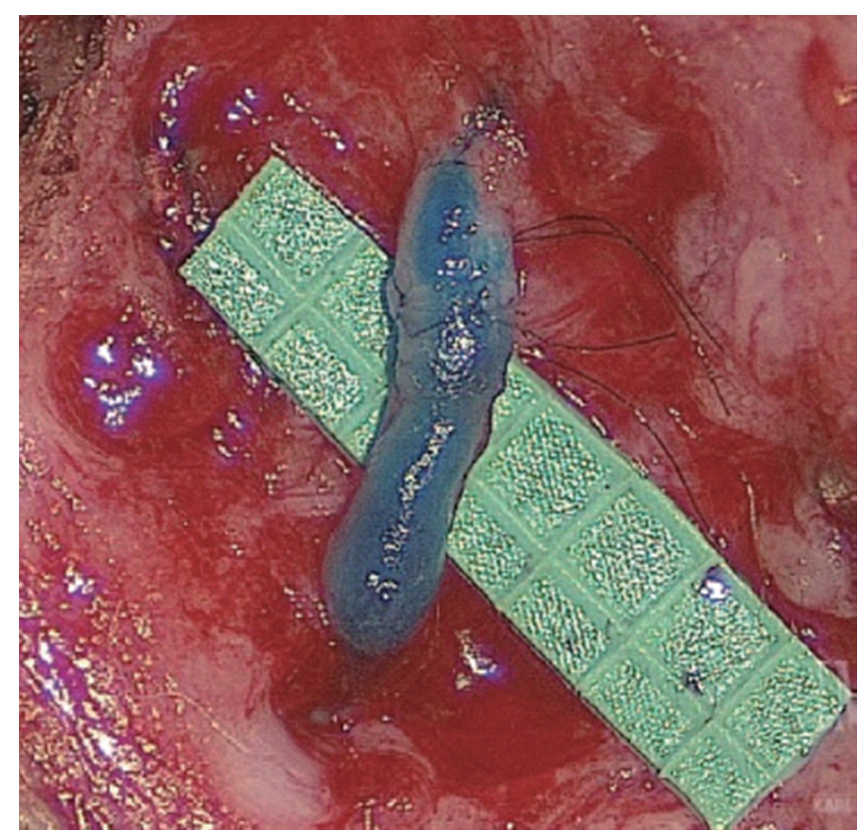

Fig. 2. The patency of the bypass is confirmed by observing the isosulfan blue dye pass from the lymphatic throught the anastamosis into the venule. Alternatively, indocyanine green may be also be used if the operating microscope has capability for injected indocyanine green flouroscopy. (1 grid=1 $\mathrm{mm}$ ) Adapted from Chang et al. [15], with permission from Wolters Kluwer Health, Inc.

The ICG lymphangiography is useful for a successful LVB. Although LVB can be done without ICG lymphangiography, it is more difficult to find functioning lymphatics suitable for LVB and to evaluate successful anastomosis and patency (Fig. 2) $[15,17]$.

The effects of LVB are known to be better in the early stages, in the upper extremities, and the secondary lymphedema. In the upper extremity, it was reported that a decrease of $11.7 \%$ of the preoperative volume excess was observed in the group of continual bandaging only, and a decrease of $47.3 \%$ of the preoperative volume excess was observed in the group of performing surgery and postoperative continual bandaging simultaneously [18]. In a prospective study, it was reported that the symptoms improved in $96 \%$ of patients and there was a quantitative improvement in $74 \%$ of patients. Moreover, the overall mean volume differential reduction was $33 \%$ at 3 months, $36 \%$ at 6 months, and $42 \%$ at 12 months after surgery [15].

\section{2) Vascularized lymph node transplant}

The indications for VLNT may include total occlusion on lymphoscintigraphy, ISL stage II with repeated episodes of cellulitis, no current cellulitis, and failure of complete deconges- tive therapy (CDT) for 6 months [19]. In the case of fibrosis of existing lymphatics, VLNT can be a better option than LVB [20]. Combining both VLNT and LVB can be considered for the improvement of lymphedema via different mechanisms in many cases. In advanced cases, VLNT can be done as a staged procedure before or after liposuction of fibroadipose soft tissue [21,22].

VLNT replenishes lymph nodes and healthy tissues in patients with lymph nodes removed. Proximal (anatomic: e.g., axilla) or distal (non-anatomic: e.g., elbow or wrist) areas are considered as locations for transplant. If a proximal transplant is made, the removed lymph nodes and damaged lymphatics will be replaced with healthy soft tissue containing lymph nodes.

In the case of distal transfer of lymph nodes, it can be expected that the lymph nodes will be located in healthy tissue rather than scarred tissue to recover their function quickly. Also, the lymph fluid, apt to be driven to the distal area, can be absorbed actively by the "gravity effect" and "catchment effect" $[23,24]$. However, the scar can be apparent and skin grafts may be necessary to cover the lymph nodes transferred due to the insufficient surrounding tissues.

There are many options for potential donor sites for VLNT. The groin is a commonly used donor site owing to well-studied anatomy, well-concealed scar and the feasibility of combining with the abdominal based free flaps for breast reconstruction and concomitant lymphedema treatment [23,25]. The supraclavicular nodes are located between the acromial-clavicular joint and the sternal notch. The donor site scar is hidden well and the small and thin flap can be harvested. This flap has not been shown to increase the risk of iatrogenic lymphedema $[23,26,27]$. The submental lymph nodes can be harvested with small elliptical skin paddle including a good number of sizable lymph nodes. This flap is unlikely to cause iatrogenic lymphedema; however, the damage of the marginal mandibular nerve should be avoided $[25,28]$. The thoracic lymph node flap can include abundant soft tissue helping to address large recipient defects and a good number of lymph nodes. But it can cause iatrogenic lymphedema to the upper extremity $[29,30]$. The greater omental lymph nodes can be harvested using the right or left gastroepiploic artery. Numerous lymph nodes can be included and the scars can be hidden well. Although the laparoscopic approach significantly reduces the donor-site complications, there can be significant intestinal complications including peritonitis, injury to intra-abdominal organs, and bowel obstruction [31,32]. In addition to the greater omental lymph node flap, the jejunal mes- 
enteric lymph node flap is an intraabdominal donor option for VLNT avoiding iatrogenic extremity lymphedema. This flap has constant vascular anatomy, a reliable lymph node packet, and a well-concealed scar. Disadvantages include injury to viscera, adhesions, and internal hernia [23,33,34].

Compared to LVB, VLNT carries slightly higher complication rates $[24,35]$. The most serious complication is the donor site or secondary lymphedema [36]. To prevent such complications, reverse lymphatic mapping using radioisotope and ICG dye should be considered [37].

It is not easy to make a certain standard for assessing and interpreting the surgical results. Each patient's stage is different, and it is also difficult to establish a subjective or objective standard of assessment. However, the outcome can be evaluated in aspects of the excess circumference reduction $(\%)$ and absolute circumference reduction $(\mathrm{cm})$.

\section{Debulking procedures}

As lymphedema progresses, fibroadipose tissue accumulation is a predominant manifestation and characteristic skin changes may follow. In this circumstance, the effects of physiologic surgery, or conservative therapy may not be helpful and the symptoms can be improved with a suction-assisted lipectomy or direct excisions.

\section{1) Liposuction}

Liposuction should only be considered when there is no pitting. The pitting is a skin and soft tissue depression expressed in $\mathrm{mm}$ that appears by pressing strongly down on a given area with the thumb for one minute $[38,39]$. The presence of pitting means there is fluid accumulation, in which case, it may be possible to try conservative therapy, such as CDT or controlled compression therapy and/or physiologic surgeries first. In the absence of the pitting, liposuction can be considered when the excess volume is not satisfactory and the heavy arms or legs are uncomfortable. Patients considering Liposuction should be cancer-free. Patients must also be compliant on wearing compression garments for life to prevent re-accumulation of lymph after surgery and to maintain their status.

When liposuction is carried out on the limb, evidence that liposuction is damaging to lymphatics is not clear [40]. Besides, studies using the lymphoscintigraphy reported no further deterioration of lymphatic function following liposuction [41]. Moreover, there are reports of an increase in lymph fluid transport and improvement in the skin blood flow to near-normal levels, these findings may provide explanations of the significant decrease in cellulitis after liposuction $[42,43]$. However, the mandatory wearing of compression garments maintaining the new equilibrium for the rest of life must be hard and cumbersome for patients.

Liposuction is a safe surgical procedure. There are several numbers of studies that report no complications during or after surgery [42,44-46]. Excessive bleeding during the surgery can occur but the possibility can be reduced by using tumescent solution and tourniquet $[39,47]$. Sensory changes or minor wound problems may occur, but most of them improve over time.

Significant post-liposuction in upper extremity volume can be achieved [42]. When patients are compliant in wearing postoperative compression garments, volume changes can be maintained for many years. Also, patients who underwent liposuction can experience a significant decrease in the number of cellulitis $[48,49]$. In the aspects of quality of life, overall well-being was improved and postoperative depression and anxiety decreased $[42,44,46]$.

\section{CONCLUSION}

In early-stage lymphedema, where protein-rich fluid accumulates, expressed as pitting, conservative treatments, such as CDT, and/or LVB should be considered. Once the stage is more advanced and skin fibrosis and adipose tissue deposition are appearing, it may be considered to attempt VLNT alone or VLNT and LVB simultaneously. If the stage is more advanced and fibroadipose tissue accumulation is dominant, then it can be considered to perform liposuction. A direct excision can be a helpful treatment option for the most advanced stage of lymphedema. Based on this review, different strategic approaches are needed according to the stage.

\section{CONFLICTS OF INTEREST}

No potential conflict of interest relevant to this article was reported.

\section{ORCID}

Byung-Joon Jeon https://orcid.org/0000-0001-6809-9439

David W. Chang https://orcid.org/0000-0002-7106-7945

\section{AUTHOR CONTRIBUTIONS}

Conception or design: BJJ, DWC. 
Acquisition, analysis, or interpretation of data: BJJ.

Drafting the work or revising: BJJ, DWC.

Final approval of the manuscript: BJJ, DWC.

\section{REFERENCES}

1. Grada AA, Phillips TJ. Lymphedema: pathophysiology and clinical manifestations. J Am Acad Dermatol 2017;77: 1009-20.

2. Kerchner K, Fleischer A, Yosipovitch G. Lower extremity lymphedema update: pathophysiology, diagnosis, and treatment guidelines. J Am Acad Dermatol 2008;59:32431.

3. Hayes S, Cornish B, Newman B. Comparison of methods to diagnose lymphoedema among breast cancer survivors: 6-month follow-up. Breast Cancer Res Treat 2005; 89:221-6.

4. Rockson SG. Lymphedema after breast cancer treatment. N Engl J Med 2018;379:1937-44.

5. Iyigun ZE, Duymaz T, Ilgun AS, Alco G, Ordu C, Sarsenov D, et al. Preoperative lymphedema-related risk factors in early-stage breast cancer. Lymphat Res Biol 2018;16:2835.

6. Amore M, Tapia L, Pattarone GR, Mercado D. Applied anatomy. In: Neligan PC, Masia J, Piller NB, editors. Lymphedema: complete medical and surgical management. New York (NY): Thieme Verlagsgruppe; 2016. p. 97-112.

7. Suami H, Scaglioni MF. Anatomy of the lymphatic system and the lymphosome concept with reference to lymphedema. Semin Plast Surg 2018;32:5-11.

8. Suami H, Pan WR, Mann GB, Taylor GI. The lymphatic anatomy of the breast and its implications for sentinel lymph node biopsy: a human cadaver study. Ann Surg Oncol 2008;15:863-71.

9. Leak LV. Electron microscopic observations on lymphatic capillaries and the structural components of the connective tissue-lymph interface. Microvasc Res 1970;2:361-91.

10. Suami H. Lymphosome concept: anatomical study of the lymphatic system. J Surg Oncol 2017;115:13-7.

11. Greene AK, Goss JA. Diagnosis and staging of lymphedema. Semin Plast Surg 2018;32:12-6.

12. Mihara M, Hara H, Araki J, Kikuchi K, Narushima M, Yamamoto $\mathrm{T}$, et al. Indocyanine green (ICG) lymphography is superior to lymphoscintigraphy for diagnostic imaging of early lymphedema of the upper limbs. PLoS One 2012; 7:e38182.

13. Ogata F, Narushima M, Mihara M, Azuma R, Morimoto Y,
Koshima I. Intraoperative lymphography using indocyanine green dye for near-infrared fluorescence labeling in lymphedema. Ann Plast Surg 2007;59:180-4.

14. Executive Committee. The diagnosis and treatment of peripheral lymphedema: 2016 consensus document of the International Society of Lymphology. Lymphology 2016;49:170-84.

15. Chang DW, Suami H, Skoracki R. A prospective analysis of 100 consecutive lymphovenous bypass cases for treatment of extremity lymphedema. Plast Reconstr Surg 2013;132:1305-14.

16. Schaverien MV, Coroneos CJ. Surgical treatment of lymphedema. Plast Reconstr Surg 2019;144:738-58.

17. Seki Y, Kajikawa A, Yamamoto T, Takeuchi T, Terashima T, Kurogi N. Single lymphaticovenular anastomosis for early-stage lower extremity lymphedema treated by the superior-edge-of-the-knee incision method. Plast Reconstr Surg Glob Open 2018;6:e1679.

18. Koshima I, Inagawa K, Urushibara K, Moriguchi T. Supermicrosurgical lymphaticovenular anastomosis for the treatment of lymphedema in the upper extremities. J Reconstr Microsurg 2000;16:437-42.

19. Cheng MH, Chen SC, Henry SL, Tan BK, Lin MC, Huang JJ. Vascularized groin lymph node flap transfer for postmastectomy upper limb lymphedema: flap anatomy, recipient sites, and outcomes. Plast Reconstr Surg 2013;131: 1286-98.

20. Hara H, Mihara M, Seki Y, Todokoro T, lida T, Koshima I. Comparison of indocyanine green lymphographic findings with the conditions of collecting lymphatic vessels of limbs in patients with lymphedema. Plast Reconstr Surg 2013;132:1612-8.

21. Granzow JW, Soderberg JM, Dauphine C. A novel twostage surgical approach to treat chronic lymphedema. Breast J 2014;20:420-2.

22. Nicoli F, Constantinides J, Ciudad P, Sapountzis S, Kiranantawat K, Lazzeri D, et al. Free lymph node flap transfer and laser-assisted liposuction: a combined technique for the treatment of moderate upper limb lymphedema. Lasers Med Sci 2015;30:1377-85.

23. Schaverien MV, Badash I, Patel KM, Selber JC, Cheng MH. Vascularized lymph node transfer for lymphedema. Semin Plast Surg 2018;32:28-35.

24. Silva AK, Chang DW. Vascularized lymph node transfer and lymphovenous bypass: novel treatment strategies for symptomatic lymphedema. J Surg Oncol 2016;113:932-9.

25. Pappalardo M, Patel K, Cheng MH. Vascularized lymph 
node transfer for treatment of extremity lymphedema: an overview of current controversies regarding donor sites, recipient sites and outcomes. J Surg Oncol 2018; 117:1420-31.

26. Maldonado AA, Chen R, Chang DW. The use of supraclavicular free flap with vascularized lymph node transfer for treatment of lymphedema: a prospective study of 100 consecutive cases. J Surg Oncol 2017;115:68-71.

27. Ooi AS, Chang DW. 5-Step harvest of supraclavicular lymph nodes as vascularized free tissue transfer for treatment of lymphedema. J Surg Oncol 2017;115:63-7.

28. Cheng MH, Huang JJ, Nguyen DH, Saint-Cyr M, Zenn MR, Tan BK, et al. A novel approach to the treatment of lower extremity lymphedema by transferring a vascularized submental lymph node flap to the ankle. Gynecol Oncol 2012;126:93-8.

29. Barreiro GC, Baptista RR, Kasai KE, dos Anjos DM, Busnardo Fde F, Modolin M, et al. Lymph fasciocutaneous lateral thoracic artery flap: anatomical study and clinical use. J Reconstr Microsurg 2014;30:389-96.

30. Raju A, Chang DW. Vascularized lymph node transfer for treatment of lymphedema: a comprehensive literature review. Ann Surg 2015;261:1013-23.

31. Ciudad P, Maruccia M, Socas J, Lee MH, Chung KP, Constantinescu T, et al. The laparoscopic right gastroepiploic lymph node flap transfer for upper and lower limb lymphedema: technique and outcomes. Microsurgery 2017;37:197-205.

32. Agko M, Ciudad P, Chen HC. Histo-anatomical basis of the gastroepiploic vascularized lymph node flap: the overlooked "micro" lymph nodes. J Plast Reconstr Aesthet Surg 2018;71:118-20.

33. Schaverien MV, Hofstetter WL, Selber JC. Vascularized jejunal mesenteric lymph node transfer for lymphedema: a novel approach. Plast Reconstr Surg 2018;141:468e-9e.

34. Coriddi M, Wee C, Meyerson J, Eiferman D, Skoracki R. Vascularized jejunal mesenteric lymph node transfer: a novel surgical treatment for extremity lymphedema. J Am Coll Surg 2017;225:650-7.

35. Basta MN, Gao LL, Wu LC. Operative treatment of peripheral lymphedema: a systematic meta-analysis of the efficacy and safety of lymphovenous microsurgery and tissue transplantation. Plast Reconstr Surg 2014;133:90513.

36. Viitanen TP, Maki MT, Seppanen MP, Suominen EA, Saaristo AM. Donor-site lymphatic function after microvascular lymph node transfer. Plast Reconstr Surg 2012;130:1246-53.
37. Dayan JH, Dayan E, Smith ML. Reverse lymphatic mapping: a new technique for maximizing safety in vascularized lymph node transfer. Plast Reconstr Surg 2015;135: 277-85.

38. Brorson H. Liposuction in arm lymphedema treatment. Scand J Surg 2003;92:287-95.

39. Schaverien MV, Munnoch DA, Brorson H. Liposuction treatment of lymphedema. Semin Plast Surg 2018;32:427.

40. Frick A, Hoffmann JN, Baumeister RG, Putz R. Liposuction technique and lymphatic lesions in lower legs: anatomic study to reduce risks. Plast Reconstr Surg 1999; 103:1868-75.

41. Brorson H, Svensson H, Norrgren K, Thorsson O. Liposuction reduces arm lymphedema without significantly altering the already impaired lymph transport. Lymphology 1998;31:156-72.

42. Boyages J, Kastanias K, Koelmeyer LA, Winch CJ, Lam TC, Sherman KA, et al. Liposuction for advanced lymphedema: a multidisciplinary approach for complete reduction of arm and leg swelling. Ann Surg Oncol 2015;22 Suppl 3: S1263-70.

43. Brorson H, Svensson H. Skin blood flow of the lymphedematous arm before and after liposuction. Lymphology 1997;30:165-72.

44. Brorson H, Ohlin K, Olsson G, Langstrom G, Wiklund I, Svensson H. Quality of life following liposuction and conservative treatment of arm lymphedema. Lymphology 2006;39:8-25.

45. Damstra RJ, Voesten HG, Klinkert P, Brorson H. Circumferential suction-assisted lipectomy for lymphoedema after surgery for breast cancer. Br J Surg 2009;96:859-64.

46. Schaverien MV, Munro KJ, Baker PA, Munnoch DA. Liposuction for chronic lymphoedema of the upper limb: 5 years of experience. J Plast Reconstr Aesthet Surg 2012; 65:935-42

47. Wojnikow S, Malm J, Brorson H. Use of a tourniquet with and without adrenaline reduces blood loss during liposuction for lymphoedema of the arm. Scand J Plast Reconstr Surg Hand Surg 2007;41:243-9.

48. Brorson H, Svensson H. Liposuction combined with controlled compression therapy reduces arm lymphedema more effectively than controlled compression therapy alone. Plast Reconstr Surg 1998;102:1058-68.

49. Lee D, Piller N, Hoffner M, Manjer J, Brorson H. Liposuction of postmastectomy arm lymphedema decreases the incidence of erysipelas. Lymphology 2016;49:85-92. 